\title{
Nifedipine treatment reduces brain damage after transient focal ischemia, possibly through its antioxidative effects
}

\author{
Mayumi Yamato ${ }^{1,2}$, Takeshi Shiba ${ }^{3}$, Tomomi Ide ${ }^{4}$, Youhei Honda ${ }^{3}$, Ken-ichi Yamada ${ }^{3}$ and Hiroyuki Tsutsui ${ }^{5}$ \\ Stroke is a major cause of mortality and morbidity in hypertensive patients. This study investigated the effects of nifedipine, \\ an L-type voltage-gated $\mathrm{Ca}^{2+}$ channel blocker, on ischemic lesion volume after focal cerebral ischemia and reperfusion in \\ rats. Rats were subjected to $1 \mathrm{~h}$ of transient middle cerebral artery occlusion (MCAO). At 2 days after MCAO, the rats were \\ randomized into two groups that were fed either a normal control diet $(n=10)$ or a nifedipine $(0.001 \%)$ containing diet $(n=11)$ \\ for 2 weeks. Nifedipine treatment significantly reduced ischemic lesion volume $\left(116.5 \pm 10.8 \mathrm{vs} .80 .0 \pm 8.2 \mathrm{~mm}^{3}, P<0.05\right)$ \\ without affecting body weight or blood pressure. It also decreased thiobarbituric-reactive substances, an index of lipid peroxide, \\ (2.6 \pm 0.4 vs. $1.7 \pm 0.1 \mu \mathrm{mol} \mathrm{g}^{-1}$ tissue, $\left.P<0.05\right)$ and increased glutathione peroxidase $\left(54.9 \pm 4.7\right.$ vs. $70.9 \pm 6.4 \mathrm{U} \mathrm{g}^{-1}$ \\ protein, $P<0.05)$ and glutathione reductase activities $\left(32.4 \pm 1.4\right.$ vs. $39.9 \pm 2.7 \mathrm{U} \mathrm{g}^{-1}$ protein, $\left.P<0.05\right)$ in the mitochondria \\ from the ischemic hemispheres. These results suggest that nifedipine treatment can reduce ischemic lesion volume after \\ focal cerebral ischemia, possibly because of the decrease in oxidative stress with an increase in antioxidant activities within \\ the ischemic area.
}

Hypertension Research (2011) 34, 840-845; doi:10.1038/hr.2011.51; published online 12 May 2011

Keywords: calcium channel blocker; free radical; ischemia reperfusion; nifedipine; stroke

\section{INTRODUCTION}

Stroke is one of the major causes of mortality and morbidity in patients with hypertension. Calcium channel blockers (CCBs) have been widely used in the treatment of hypertension. A large scale clinical study, the Antihypertensive and Lipid-Lowering Treatment to Prevent Heart Attack Trial, reported that CCBs could prevent stroke better than diuretics with a similar blood-pressure-lowering effect. ${ }^{1}$

Oxidative stress has an important role in the pathogenesis of ischemic brain damage. ${ }^{2-4}$ During ischemia, a robust influx of calcium $\left(\mathrm{Ca}^{2+}\right)$ activates glutamate receptors that induce the generation of reactive oxygen species from mitochondria ${ }^{5}$ and reactive nitrogen species, ${ }^{6}$ leading to lipid peroxidation. ${ }^{7}$ L-type $\mathrm{Ca}^{2+}$ channels and $\mathrm{N}$-methyl-D-aspartate receptors have a crucial role in the entry of $\mathrm{Ca}^{2+}$ into neurons. ${ }^{8}$ Thus, these results suggest that the oxidative stress associated with ischemic brain damage can be ameliorated by the blockade of $\mathrm{Ca}^{2+}$ entry via CCBs. In fact, previous experimental studies have demonstrated that CCBs could reduce stroke size and neurological deficits after focal brain ischemia. ${ }^{9-12}$ Lukic-Panin et al. ${ }^{10}$ demonstrated that CCBs reduced oxidative stress and infarct volume after transient middle cerebral artery occlusion (MCAO) in rats. These findings suggest that CCBs have favorable neuroprotective effects against stroke. However, in previous studies, the treatment of experimental animals with CCBs was started before the onset of ischemia. The effects of CCBs on ischemic lesion size have not been examined with administration after the onset of cerebral ischemia.

Therefore, the aim of this study was to determine the effects of nifedipine treatment, when started after the ischemic insult, on focal brain ischemia induced by transient MCAO in rats. We also determined its effect on oxidative stress and antioxidant enzymes in the brain.

\section{METHODS}

Animal models

Male Wistar rats (6 weeks old) were purchased from KBT Oriental (Saga, Japan). The rats were housed in a temperature- and humidity-controlled room, and were fed a commercial diet (MF; Oriental Yeast Tokyo, Japan) and water ad libitum.

Focal cerebral ischemia was created in rats by transient MCAO as previously described. ${ }^{13,14}$ In brief, the rats were anesthetized with $2 \%$ isoflurane (Dainippon Pharmaceutical, Osaka, Japan) and maintained with $1 \%$ isoflurane using a face mask. A 1-cm midline incision was made on the anterior neck, and the right common carotid artery, external carotid artery and internal carotid artery were exposed. The common carotid artery and external carotid artery were

${ }^{1}$ Department of REDOX Medicinal Science, Faculty of Pharmaceutical Sciences, Kyushu University, Fukuoka, Japan; ${ }^{2}$ Medical Redox Imaging Group, Innovation Center for Medical Redox Navigation, Kyushu University, Fukuoka, Japan; ${ }^{3}$ Department of Bio-function Science, Faculty of Pharmaceutical Sciences, Kyushu University, Fukuoka, Japan; ${ }^{4}$ Department of Cardiovascular Medicine, Graduate School of Medical Sciences, Kyushu University, Fukuoka, Japan and ${ }^{5}$ Department of Cardiovascular Medicine, Hokkaido University Graduate School of Medicine, Sapporo, Japan

Correspondence: Dr M Yamato, Medical Redox Imaging Group, Innovation Center for Medical Redox Navigation, Kyushu University, Fukuoka 812-8582, Japan.

E-mail: yamato68@redoxnavi.med.kyushu-u.ac.jp

Received 15 October 2010; revised 28 January 2011; accepted 7 February 2011; published online 12 May 2011 
ligated, and a suture was placed around the internal carotid artery for ligation. A thrombus was created by inserting a $4-0$ nylon surgical thread into the internal carotid artery via a small incision. The middle cerebral artery was occluded by advancing the thrombus into the internal carotid artery to block the origin of the middle cerebral artery. After $1 \mathrm{~h}$ of MCAO, the middle cerebral artery was reperfused by withdrawing the thrombus. During these procedures, the rectal temperature was maintained at $37^{\circ} \mathrm{C}$ by placing the rat on a heating pad.

In our preliminary experiment, the changes in oxidative damage and antioxidant enzymes were assessed in the rat brain before the procedure $(n=6)$ at $1 \mathrm{~h}$ after MCAO $(n=6)$ and at 2 days after MCAO and reperfusion $(n=6)$. To examine the effects of the CCBs on brain damage after transient focal ischemia, all surviving rats were randomly assigned to two groups at 2 days after MCAO, control diet ( $n=18$, MF; Oriental Yeast) and $0.001 \%$ nifedipineadded MF diet ( $n=18$, Oriental Yeast), for 2 weeks. The amount of nifedipine intake was estimated to be $\sim 0.8 \mathrm{mg} \mathrm{kg}^{-1}$ body weight per day based on the amount of food intake in our animals. All procedures and animal care were approved by the Animal Care and Use Committee, Kyushu University, and carried out in accordance with the Guidelines for Animal Experiments, Kyushu University.

\section{Animal characteristics}

Arterial pressure and heart rate were analyzed by a Power Lab 4/30 with a blood pressure transducer (MLT0670, AD Instruments Japan, Nagoya, Japan). Arterial $\mathrm{pH}, \mathrm{pO}_{2}$ and $\mathrm{pCO}_{2}$ levels were analyzed by a GASTAT-mini (Techno Medica, Yokohama, Japan).

\section{Estimation of ischemic lesion volume by MRI}

Ischemic lesion volume was estimated using a 0.4 T MRI (Hitachi Medical Corporation, Tokyo, Japan) and a standard T2-weighted fast spin echo sequence (Repetition time/Echo time/Flip angle, $3000 \mathrm{~ms} / 90 \mathrm{~ms} / 90$-degree, for 2.5-mm-thick slices) as previously described. ${ }^{15-17}$ Briefly, after anesthesia was induced with pentobarbital (Dainabot, Tokyo, Japan), the rats were placed on a non-magnetic holder, which was placed into the bore of the MRI magnet. Spin-echo scout images were acquired first in the coronal and then in the sagittal planes to select reproducible coronal slice positions. A total of 12 contiguous coronal slice images were obtained from each rat. The ischemic lesions in T2-weighted imaging were defined as hyperintense areas on the image. To measure the ischemic lesion volume, the outline of the hyperintensive area was traced on the MRI, and then the sum of the areas gave the volume of the ischemic lesion volume in $\mathrm{mm}^{3}$.

\section{Measurement of brain water content}

Immediately after the rats were killed, the brain was removed, and the cerebrum was divided into ischemic and contralateral hemispheres. The wet weights of the tissue segments were measured. After segments were dried in a desiccating oven for $24 \mathrm{~h}$ at $100{ }^{\circ} \mathrm{C}$, their dry weights were measured, and the percentage water content of the tissues was calculated.

Measurement of brain thiobarbituric-reactive substances (TBARSs) The concentration of TBARSs was measured in both the ischemic and the contralateral hemispheres. After MRI experiments, the animals were killed by transcardiac perfusion with heparinized saline, and the brains were removed. The brain was homogenized $(3: 7, \mathrm{w} / \mathrm{v})$ in $1.15 \%$ of $\mathrm{KCl}$ and $5 \mathrm{~mm}$ of 2,6-di- $t$ butyl-4-methyphenol. A $0.1-\mathrm{ml}$ sample of the homogenate was mixed with reagents to give a final concentration of $2 \mathrm{~mm}$ EDTA, $7.5 \%$ acetic acid and $0.4 \%$ SDS and then reacted with $0.3 \%$ thiobarbituric acid (Wako Pure Chemical Industries, Osaka, Japan) in a boiling water bath for $45 \mathrm{~min}$. After being cooled, the chromogen was extracted in $n$-butanol:pyridine $(15: 1, \mathrm{v} / \mathrm{v})$. The TBARS concentration was calculated from the absorption values of the butanolextracted supernatant at $532 \mathrm{~nm}$. 1,1,3,3-Tetraethoxypropane was used as the standard.

\section{Measurement of brain antioxidant enzymes}

Mitochondrial and cytoplasmic fractions were isolated from both ischemic and contralateral hemispheres as previously described. ${ }^{14}$ Glutathione peroxidase
(GPx) and glutathione reductase (GR) activities in each fraction were measured according to the method of Yamamoto and Takahashi ${ }^{18}$ and Carlberg et al., ${ }^{19}$ respectively. One unit of both enzyme activities was defined as the oxidation of $1 \mu \mathrm{mol}$ of nicotinamide adenine dinucleotide phosphate per minute by measuring the absorbance measured at $340 \mathrm{~nm}$. The superoxide dismutase (SOD) activity was determined by monitoring the inhibition of xanthine oxidase-mediated cytochrome $c$ reduction from the absorbance at $550 \mathrm{~nm} \cdot .^{20,21}$ The specific activity was expressed as units per gram of protein.

\section{Measurement of mitochondrial complex activities}

Mitochondrial fractions were isolated from both ischemic and contralateral hemispheres. The specific activity of the mitochondrial complex enzymes, including complexes I, II, III and IV, was measured in the mitochondria isolated from the whole brains of each group as previously described.22 The enzymatic activities were expressed as units per gram of protein.

\section{Statistical analysis}

All of the results are shown as the mean \pm s.e.m. Statistical significance was analyzed using the two-tailed $t$-test or Tukey-Kramer test. A probability value of 0.05 was set as the minimum level of statistical significance.

\section{RESULTS}

TBARS were significantly increased in the ischemic hemispheres compared with the contralateral hemispheres from rats at 2 days after MCAO and reperfusion (Figure 1). The GR activities in the mitochondrial fraction were significantly decreased in the ischemic hemispheres compared with the contralateral hemispheres from rats at $1 \mathrm{~h}$ after MCAO and at 2 days after MCAO and reperfusion (Figure 2a). The GPx and SOD activities in the mitochondrial fraction did not change in either group (Figure 2a). The GPx and GR activities in the cytoplasmic fraction from the ischemic hemisphere were significantly decreased in the ischemic hemispheres compared with the contralateral hemispheres from rats at $1 \mathrm{~h}$ after MCAO and at 2 days after MCAO and reperfusion (Figure 2b). The SOD activity tended to be lower in the cytoplasm from the ischemic hemispheres compared with the contralateral hemispheres from rats at $1 \mathrm{~h} \mathrm{MCAO}$ and at 2 days after MCAO and reperfusion; this difference, however, did not reach statistical significance (Figure $2 b$ ).

There were no significant differences in body weight and food intake between control and nifedipine-treated rats. The mean arterial pressure, heart rate and blood gases were also comparable between the

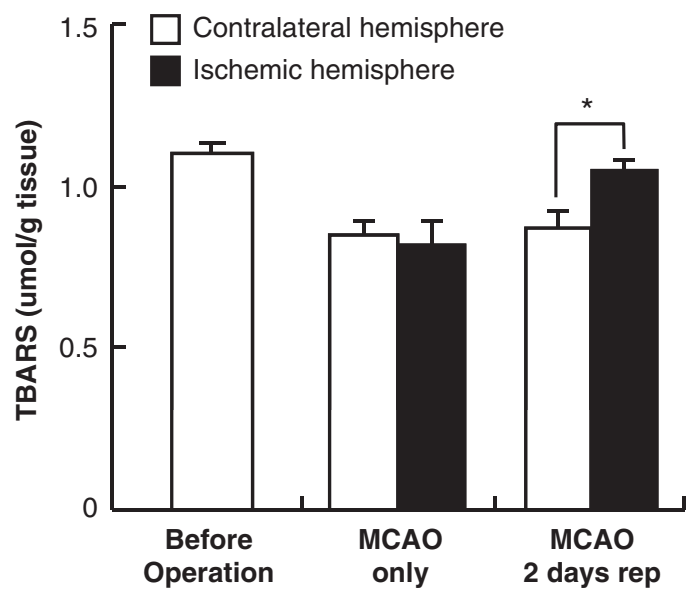

Figure 1 TBARS in ischemic (closed bars) and contralateral hemispheres (open bars) from rats before the operation $(n=6)$ at $1 \mathrm{~h}$ after MCAO $(n=6)$ and at 2 days after MCAO and reperfusion $(n=6)$. TBARS of the ischemic hemisphere were significantly increased in the rats at 2 days after MCAO and reperfusion. ${ }^{*} P<0.05$ vs. the contralateral hemisphere. 

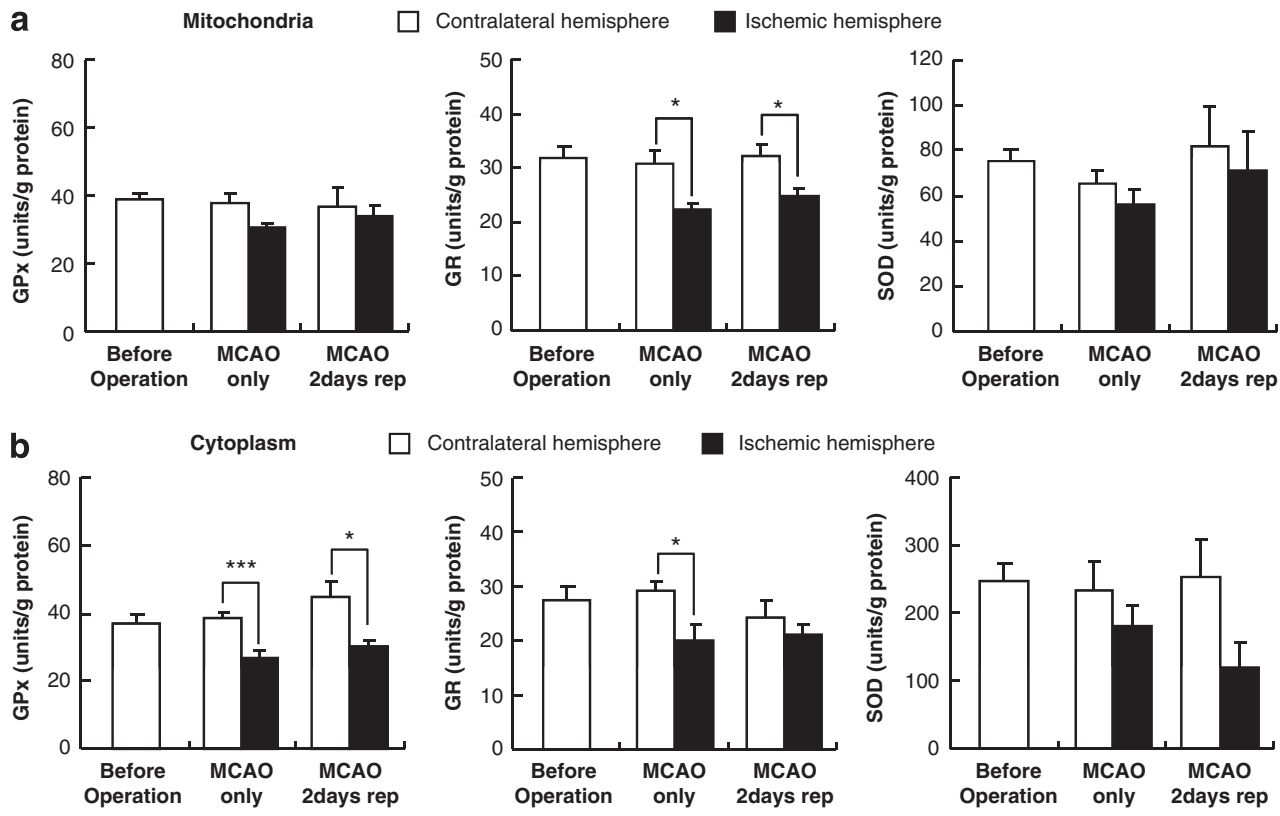

Figure 2 The GPx, GR and SOD activities in the mitochondrial (a) and cytoplasmic fractions (b) of the contralateral (open bars) and ischemic hemispheres (closed bras) from the rats before the operation $(n=6)$ at $1 \mathrm{~h}$ after MCAO $(n=6)$ and at 2 days after MCAO and reperfusion $(n=6)$. ${ }^{*} P<0.05$ and $* * * P<0.005 v s$. the contralateral hemisphere in each group of rats.

Table 1 Animal charactaristics

\begin{tabular}{lcc}
\hline & Control $(\mathrm{n}=10)$ & Nifedipine $(\mathrm{n}=11)$ \\
\hline Body weight (g) & $311.3 \pm 14.9$ & $292.6 \pm 10.4$ \\
Food intake ( $\mathrm{g} \mathrm{kg}^{-1}$ body weight per day) & $82.8 \pm 4.7$ & $78.9 \pm 2.6$ \\
Mean arterial pressure (mm Hg) & $97.4 \pm 1.6$ & $97.1 \pm 2.3$ \\
Heart rate (b.p.m.) & $395 \pm 20$ & $396 \pm 11$ \\
$\mathrm{pH}$ & $7.4 \pm 0.02$ & $7.4 \pm 0.01$ \\
$\mathrm{pO}_{2}(\mathrm{~mm} \mathrm{Hg})$ & $106.5 \pm 7.4$ & $107.5 \pm 6.6$ \\
$\mathrm{pCO}_{2}(\mathrm{~mm} \mathrm{Hg})$ & $41.5 \pm 4.7$ & $42.3 \pm 5.5$ \\
\hline
\end{tabular}

Each value represents the mean \pm s.e.m. The data were obtained at 2 weeks after treatment.

groups (Table 1), indicating that nifedipine did not exert significant hypotensive effects in this study.

Ischemic lesions were visualized by T2-weighted MRI. Ischemic lesion volume was significantly smaller in nifedipine-treated rats compared with control rats by $30 \%$ (Figures $3 \mathrm{a}$ and $\mathrm{b}$ ). Water content was also lower in the ischemic hemisphere of nifedipine-treated rats compared with that of control rats (Figure 3c).

TBARS were significantly increased in the ischemic hemispheres compared with the levels in the contralateral hemispheres from control rats. TBARS in the ischemic hemispheres from nifedipinetreated rats were significantly lower compared with those from control rats and were comparable with those in the contralateral hemispheres (Figure 4).

The GPx and GR activities in the mitochondrial fraction were comparable between ischemic and contralateral hemispheres from control rats, whereas those in the mitochondrial fraction of the ischemic hemispheres were significantly increased compared with those in the contralateral hemispheres from nifedipine-treated rats (Figure 5a). The SOD activity tended to be lower in the mitochondrial fraction from the ischemic hemispheres compared with the contralateral hemispheres, but this difference did not reach statistical significance (Figure 5a). The GPx activity in the cytoplasmic fraction from the ischemic hemispheres was significantly increased compared with that in the contralateral hemispheres of the control group, and this increase was similarly observed in the nifedipine-treated rats (Figure 5b). The GR and SOD activities in the cytoplasm did not change in either group (Figure 5b).

We measured the mitochondrial complex activities to estimate mitochondrial function, but these enzyme activities did not change in either group (Figure 6).

\section{DISCUSSION}

This study demonstrated that treatment with nifedipine, initiated even after the onset of ischemia, could reduce ischemic lesion volume after transient MCAO in association with the reduction in oxidative stress and the increase in mitochondrial GPx and GR activities in the ischemic brain.

Oxidative stress has an important role in the pathogenesis of ischemic brain damage. ${ }^{2-4}$ We have also demonstrated the accumulation of lipid peroxidation products and the decrease in antioxidative enzymes in the ischemic hemispheres in our model at 2 days after MCAO and reperfusion. Previous studies have already reported the direct neuroprotective effects of CCBs during the early phase of ischemia reperfusion. ${ }^{9-12}$ This study has extended these findings and demonstrated for the first time that nifedipine can ameliorate brain damage even when administered after reperfusion. These findings might also suggest that the intracellular $\mathrm{Ca}^{2+}$ overload, which is probably because of the excessive $\mathrm{Ca}^{2+}$ entry through overactivated glutamate receptors, is a crucial event not only during the early phase of ischemia but also during the process of persistent brain damage. Neuronal $\mathrm{Ca}^{2+}$ overload may trigger the formation of intracellular reactive oxygen species. ${ }^{23}$ Moreover, hydrogen peroxide has been shown to enhance the $\mathrm{Ca}^{2+}$ current through L-type $\mathrm{Ca}^{2+}$ channels in cultured rat dentate granule cells. ${ }^{24}$ The vicious circle between ischemia, $\mathrm{Ca}^{2+}$ overload and oxidative stress may be involved in the ischemic brain damage, and cause further deterioration in the integ- 
a
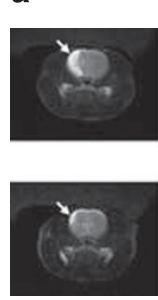

Control

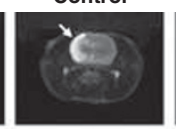

Nifedipine

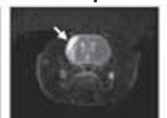

b

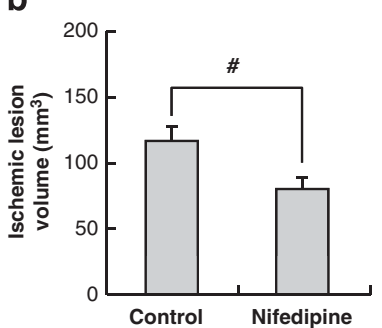

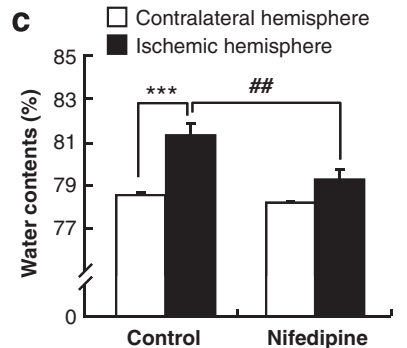

Figure $3 \mathrm{MRI}$ images of ischemic lesion volume (a), summary data of ischemic lesion volume using MRI (b), and water content of the brains (c) in control and nifedipine-treated rats. Ischemic lesion volume and water content were significantly smaller in nifedipine-treated rats. $\#<0.05$ vs. control rats. ${ }_{* * *} P<0.005$ vs. the contralateral hemisphere. ${ }^{\# \# P}<0.01$ vs. the ischemic hemisphere from control rats.

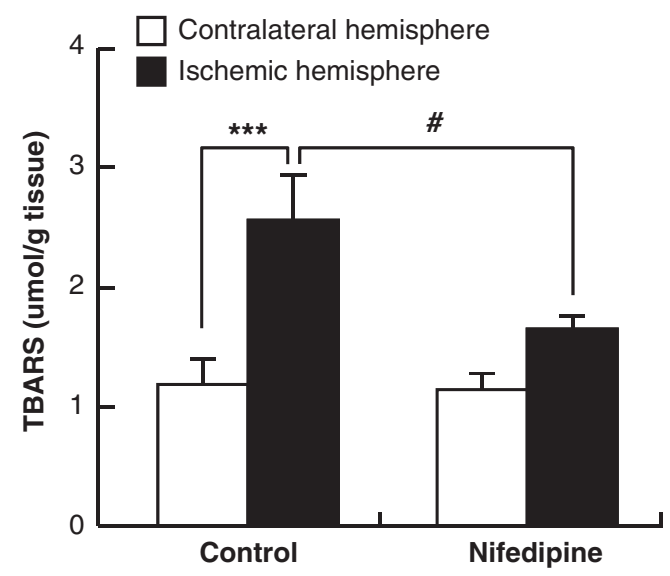

Figure 4 TBARS in ischemic (closed bars) and contralateral hemispheres (open bars) from control $(n=10)$ and nifedipine-treated rats $(n=11)$. TBARS of the ischemic hemisphere was significantly reduced in the nifedipinetreated rats compared with control rats. ${ }^{* * *} P<0.005$ vs. the contralateral hemisphere. ${ }^{\#} P<0.05$ vs. the ischemic hemisphere from control rats. rity of at-risk tissue. Therefore, CCBs may prevent these processes of $\mathrm{Ca}^{2+}$ overload and oxidative stress in the ischemic brain.

There is accumulating evidence that CCBs have antioxidative effects in the setting of lysophosphatidylcholine-induced injury, ${ }^{25}$ salt-loaded, stroke-prone spontaneous hypertension, ${ }^{26}$ atherosclerosis ${ }^{12}$ and cerebral ischemia. ${ }^{10,27}$ It has also been reported to increase the activity of antioxidant enzymes, such as GPx, GR and SOD. ${ }^{21,27,28}$ In agreement with these previous results, this study showed that nifedipine treatment inhibited lipid peroxidation and increased GPx and GR activities in the mitochondria of ischemic brains (Figures 4 and 5). The increases of antioxidant enzyme activities in the mitochondria may decrease oxidative stress because the mitochondrial electron transport chain is one of the major sources of reactive oxygen species in ischemic brains. ${ }^{29}$ In this study, SOD activities in the mitochondrial and cytoplasmic fractions of the brain were not altered (Figure 5). However, the results were not in agreement with previous studies in which SOD activity was increased by nifedipine treatment in the early phase of brain reperfusion. ${ }^{27}$ In this study, nifedipine treatment did not affect blood pressure or heart rate, indicating that the neuroprotective effects of nifedipine are independent of its blood pressurelowering effect on ischemic lesion volume.
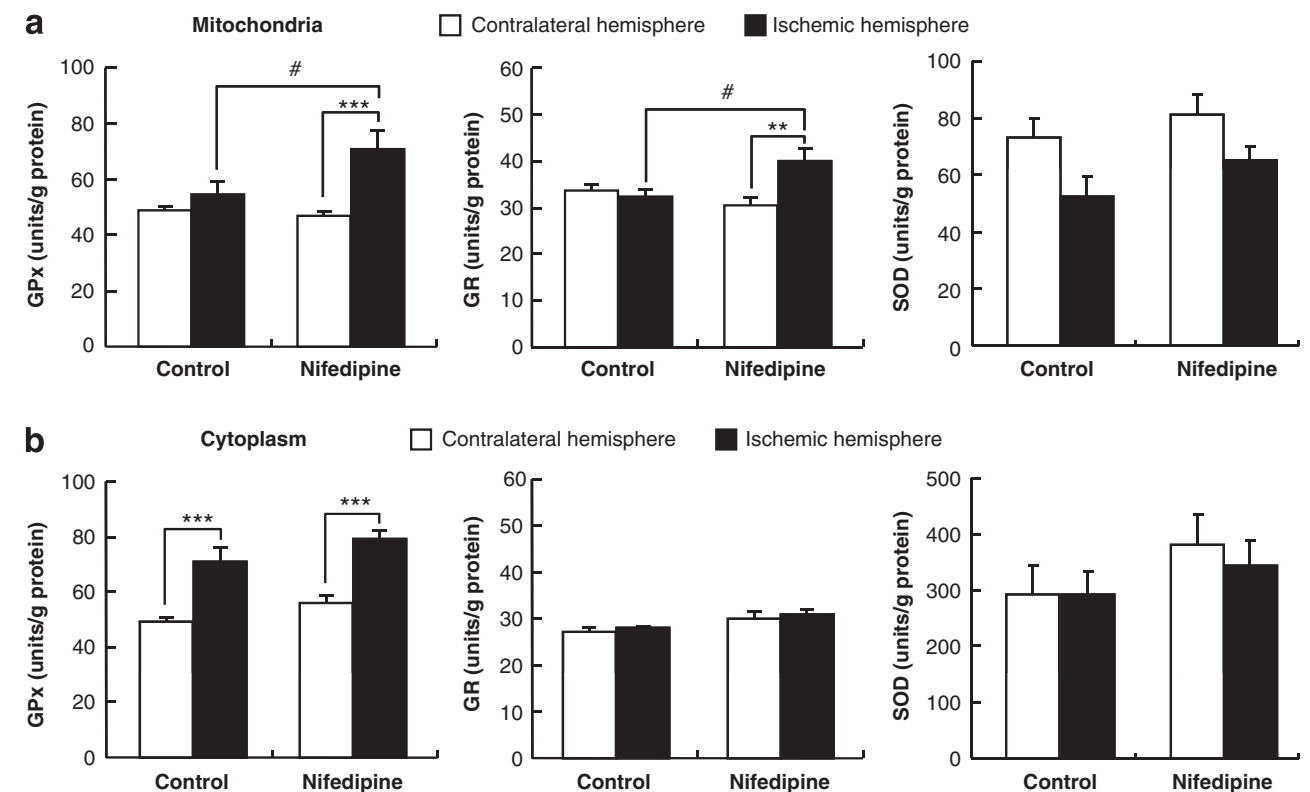

Figure $5 \mathrm{GPx}, \mathrm{GR}$ and SOD activities in the mitochondrial (a) and cytoplasmic fractions (b) of the contralateral (open bars) and ischemic hemispheres (closed bras) from control $(n=10)$ and nifedipine-treated rats $(n=11)$. ${ }^{* *} P<0.01$ and ${ }^{* * *} P<0.005$ vs. the contralateral hemisphere in each group of rats. $\# P<0.05$ vs. the ischemic hemisphere from control rats. 

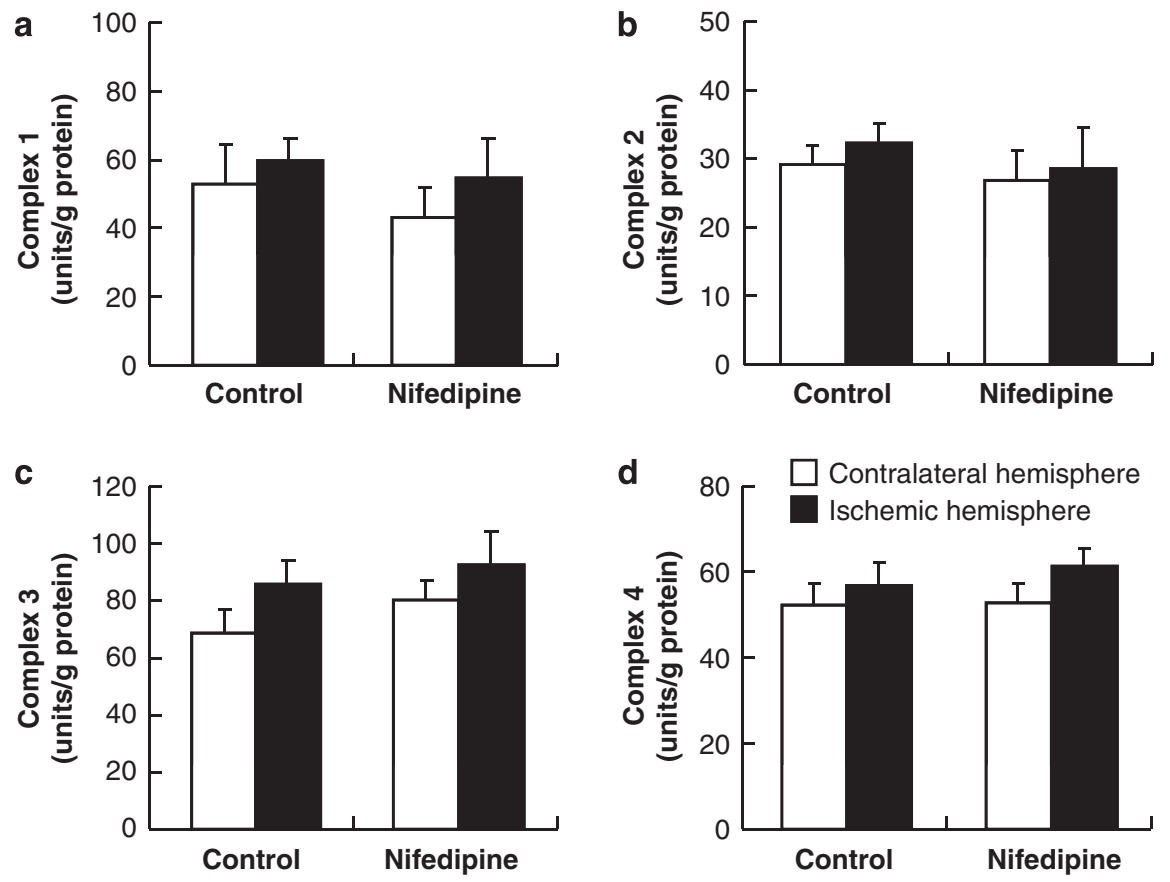

Figure 6 Mitochondrial complex activities in the contralateral (open bars) and ischemic hemispheres (closed bars) from control ( $n=10)$ and nifedipinetreated rats $(n=11)$.

In this study, we estimated the ischemic lesion volume using T2weighted MRI. Proton density in MRI indicates the density of the water proton spin; thus, the $\mathrm{T} 1$ and $\mathrm{T} 2$ of water proton spins depend on the physiochemical state of water. Many pathological changes, such as tumors, edema and hemorrhage, are associated with a change in water content. Therefore, T2-weighted MRI has been extensively applied to delineate lesions in both clinical and experimental diagnostic studies, ${ }^{30,31}$ because both $\mathrm{T} 1$ and $\mathrm{T} 2$ have been reported to correlate with tissue water content. In particular, the T2 change is shown to be larger than the T1 change. ${ }^{32,33}$ In fact, our data showed parallel changes in ischemic lesion volume and water content (Figure 3 ).

There are several limitations of this study. First, we did not measure cerebral blood flow in the ischemic and non-ischemic regions after MCAO. However, the rats were randomized after 2 days of MCAO. Therefore, the decrease in cerebral blood flow after MCAO may not differ between rats with or without nifedipine treatment. Second, we could not exclude the possibility that an increase of blood flow to the ischemic area via the improvement of endothelial function may be involved in the reduction of ischemic lesion volume in nifedipinetreated rats. Third, we have not examined whether the findings of this study can be extended to other CCBs, and we definitely need to perform additional studies to clarify this crucial issue. However, we feel that the beneficial effects of nifedipine may be observed with CCBs other than nifedipine because amlodipine and azelnidipine have been reported to inhibit apoptosis in an animal model of brain infarction due to 90 min MCAO and $24 \mathrm{~h}$ of reperfusion. ${ }^{10}$ Fourth, we have not examined whether the administration of direct antioxidants can exert similar beneficial effects on brain damage as nifedipine, which may support our findings about the mechanisms of action of nifedipine.

In conclusion, this study demonstrated that nifedipine treatment could reduce ischemic lesion volume and oxidative stress after MCAO in rats. These beneficial effects of nifedipine may be partly because of the antioxidative action via increasing antioxidative enzymes in the ischemic brain.
1 ALLHAT Officers and Coordinators for the ALLHAT Collaborative Research Group. The Antihypertensive and Lipid-Lowering Treatment to Prevent Heart Attack Trial. Major outcomes in high-risk hypertensive patients randomized to angiotensin-converting enzyme inhibitor or calcium channel blocker vs diuretic: the Antihypertensive and Lipid-Lowering Treatment to Prevent Heart Attack Trial (ALLHAT). JAMA 2002; 288: 2981-2997.

2 Huang J, Agus DB, Winfree CJ, Kiss S, Mack WJ, McTaggart RA, Choudhri TF, Kim LJ, Mocco J, Pinsky DJ, Fox WD, Israel RJ, Boyd TA, Golde DW, Connolly Jr ES. Dehydroascorbic acid, a blood-brain barrier transportable form of vitamin $\mathrm{C}$, mediates potent cerebroprotection in experimental stroke. Proc Natl Acad Sci USA 2001; 98: 11720-11724.

3 Kato N, Yanaka K, Hyodo K, Homma K, Nagase S, Nose T. Stable nitroxide Tempol ameliorates brain injury by inhibiting lipid peroxidation in a rat model of transient focal cerebral ischemia. Brain Res 2003; 979: 188-193.

4 Yamato M, Egashira T, Utsumi H. Application of in vivo ESR spectroscopy to measurement of cerebrovascular ROS generation in stroke. Free Radic Biol Med 2003; 35: 1619-1631.

5 Chinopoulos C, Adam-Vizi V. Calcium, mitochondria and oxidative stress in neuronal pathology. Novel aspects of an enduring theme. FEBS J 2006; 273: 433-450.

6 Tjong YW, Jian K, Li M, Chen M, Gao TM, Fung ML. Elevated endogenous nitric oxide increases $\mathrm{Ca} 2+$ flux via L-type $\mathrm{Ca} 2+$ channels by S-nitrosylation in rat hippocampal neurons during severe hypoxia and in vitro ischemia. Free Radic Biol Med 2007; 42: 52-63.

7 Doyle KP, Simon RP, Stenzel-Poore MP. Mechanisms of ischemic brain damage. Neuropharmacology 2008; 55: 310-318.

8 Seeburg PH. The TINS/TiPS lecture. The molecular biology of mammalian glutamate receptor channels. Trends Neurosci 1993; 16: 359-365.

9 Abe K, Kogure K, Watanabe T. Prevention of ischemic and postischemic brain edema by a novel calcium antagonist (PN200-110). J Cereb Blood Flow Metab 1988; 8: 436-439.

10 Lukic-Panin V, Kamiya T, Zhang H, Hayashi T, Tsuchiya A, Sehara Y, Deguchi K, Yamashita T, Abe K. Prevention of neuronal damage by calcium channel blockers with antioxidative effects after transient focal ischemia in rats. Brain Res 2007; 1176: 143-150.

11 Mogi M, Iwai M, Chen R, Iwanami J, Ide A, Tsukuda K, Yoshii T, Horiuchi M. Amlodipine treatment reduces stroke size in apolipoprotein E-deficient mice. Am J Hypertens 2006; 19: 1144-1149.

12 Yoshii T, Iwai M, Li Z, Chen R, Ide A, Fukunaga S, Oshita A, Mogi M, Higaki J, Horiuchi $\mathrm{M}$. Regression of atherosclerosis by amlodipine via anti-inflammatory and anti-oxidative stress actions. Hypertens Res 2006; 29: 457-466.

13 Koizumi J, Yoshida Y, Nakazawa T, Ooneda G. Experimental studies of ischemic brain edema 1. A new experimental model of cerebral embolism in rats in which recirculation can be introduced in the ischemic area. Jpn J Stroke 1986; 8: 1-8.

14 Yamato M, Shiba T, Yamada K, Watanabe T, Utsumi H. Noninvasive assessment of the brain redox status after transient middle cerebral artery occlusion using Overhauser- 
enhanced magnetic resonance imaging. J Cereb Blood Flow Metab 2009; 29: 1655-1664.

15 Kollmar R, Schabitz WR, Heiland S, Georgiadis D, Schellinger PD, Bardutzky J, Schwab S. Neuroprotective effect of delayed moderate hypothermia after focal cerebral ischemia: an MRI study. Stroke 2002; 33: 1899-1904.

16 Peeling J, Corbett D, Del Bigio MR, Hudzik TJ, Campbell TM, Palmer GC. Rat middle cerebral artery occlusion: correlations between histopathology, T2-weighted magnetic resonance imaging, and behavioral indices. J Stroke Cerebrovasc Dis 2001; 10: 166-177.

17 Schabitz WR, Schade H, Heiland S, Kollmar R, Bardutzky J, Henninger N, Müller H, Carl U, Toyokuni S, Sommer C, Schwab S. Neuroprotection by hyperbaric oxygenation after experimental focal cerebral ischemia monitored by MRI. Stroke 2004; 35: 1175-1179.

18 Yamamoto Y, Takahashi K. Glutathione peroxidase isolated from plasma reduces phospholipid hydroperoxides. Arch Biochem Biophys 1993; 305: 541-545.

19 Carlberg I, Mannervik B. Purification and characterization of the flavoenzyme glutathione reductase from rat liver. J Biol Chem 1975; 250: 5475-5480.

20 Flohe L, Otting F. Superoxide dismutase assays. Methods Enzymol 1984; 105 : 93-104.

21 Fukuo K, Yang J, Suzuki T, Kaimoto T, Takemura Y, Yasuda O, Suhara T, Sato N, Ogihara T. Nifedipine upregulates manganese superoxide dismutase expression in vascular smooth muscle cells via endothelial cell-dependent pathways. Hypertens Res 2003; 26: 503-508.

22 Trounce IA, Kim YL, Jun AS, Wallace DC. Assessment of mitochondrial oxidative phosphorylation in patient muscle biopsies, lymphoblasts, and transmitochondrial cell lines. Methods Enzymol 1996; 264: 484-509.

23 Hall ED, Andrus PK, Althaus JS, VonVoigtlander PF. Hydroxyl radical production and lipid peroxidation parallels selective post-ischemic vulnerability in gerbil brain. J Neurosci Res 1993; 34: 107-112.
24 Akaishi T, Nakazawa K, Sato K, Saito H, Ohno Y, Ito Y. Hydrogen peroxide modulates whole cell $\mathrm{Ca} 2+$ currents through L-type channels in cultured rat dentate granule cells. Neurosci Lett 2004; 356: 25-28.

25 Matsubara M, Hasegawa K. Benidipine, a dihydropyridine-calcium channel blocker, prevents lysophosphatidylcholine-induced injury and reactive oxygen species production in human aortic endothelial cells. Atherosclerosis 2005; 178: 57-66.

26 Matsubara M, Akizuki O, Ikeda J, Saeki K, Yao K, Sasaki K. Benidipine, an antihypertensive drug, inhibits reactive oxygen species production in polymorphonuclear leukocytes and oxidative stress in salt-loaded stroke-prone spontaneously hypertensive rats. Eur J Pharmacol 2008; 580: 201-213.

27 El-Abhar HS, Shaalan M, Barakat M, El-Denshary ES. Effect of melatonin and nifedipine on some antioxidant enzymes and different energy fuels in the blood and brain of global ischemic rats. J Pineal Res 2002; 33: 87-94.

28 Mohan K, Dubey ML, Ganguly NK, Kalra A, Mahajan RC. Effect of nifedipine on oxidative damage of erythrocytes in Plasmodium berghei-infected mice. Clin Immunol Immunopathol 1993; 69: 244-247.

29 Piantadosi CA, Zhang J. Mitochondrial generation of reactive oxygen species after brain ischemia in the rat. Stroke 1996; 27: 327-331; discussion 332.

30 van Bruggen N, Cullen BM, King MD, Doran M, Williams SR, Gadian DG, Cremer JE. T2- and diffusion-weighted magnetic resonance imaging of a focal ischemic lesion in rat brain. Stroke 1992; 23: 576-582.

31 van Bruggen N, Roberts TP, Cremer JE. The application of magnetic resonance imaging to the study of experimental cerebral ischaemia. Cerebrovasc Brain Metab Rev 1994; 6: $180-210$.

32 Dijkhuizen RM, Nicolay K. Magnetic resonance imaging in experimental models of brain disorders. J Cereb Blood Flow Metab 2003; 23: 1383-1402.

33 Kato H, Kogure K, Ohtomo H, Izumiyama M, Tobita M, Matsui S, Yamamoto E, Kohno H, Ikebe $Y$, Watanabe T. Characterization of experimental ischemic brain edema utilizing proton nuclear magnetic resonance imaging. J Cereb Blood Flow Metab 1986; 6: 212-221. 